\title{
Lecteurs 2012
}

Le Comité de rédaction remercie les rapporteurs et les lecteurs ayant apporté leurs concours à l'évaluation des articles publiés ou analysés en 2012 (liste au 15 novembre).

Abéla Gonzague, EDF

Ademola Janet, Helmholtz Inst. (All)

Améon Roselyne, IRSN

Ammerich Marc, CEA

Ansoborlo Eric, CEA

Aubert Cédric, IRSN

Aubert Bernard, IRSN

Aubineau-Lanièce Isabelle, CEA

Aurengo André, CHU Pitié-Salpêtrière

Badot Pierre-Marie, Univ. Franche Comté

Baglan Nicolas, CEA

Barescut Jean-Claude, IRSN

Bérard Philippe, CEA

Bertelli Luis, Los Alamos (USA)

Bertho Jean-Marc, IRSN

Blanchardon Éric, IRSN

Bochud François, CHU Lausanne (CH)

Bolch Wesley, Univ. Florida (USA)

Bonniaud Guillaume, Centre Saint-Yves

Bordy Jean-Marc, CEA

Caamano Delphine, ASN

Calvez Marianne, CEA

Cazoulat Alain, SRPA

Champlong Josette, SFRP

Corcho José, BAG $(\mathrm{CH})$

Cossonnet Catherine, IRSN

De Cock Jean-Paul, Electrabel (B)

De Vita Antoine, AREVA

Decossas Jean-Louis, PEARL Limoges

Doumont Pierre, Electrabel (B)

Etard Cécile, IRSN

Etherington George, HPA (UK)
Farah Jad, CHU Pitié-Salpêtrière

Faure Sophie, CEA

Fournier Marc, ASN

Franck Didier, IRSN

Frelon Sandrine, IRSN

Gauron Christine, INRS

Georges Jean-Louis, Hôpital Versailles

Gouronnec Anne-Marie, CEA

Gschwind Régine, Univ. Franche Comté

Hall Janet, Institut Curie

Hémidy Pierre-Yves, EDF

Henge Marie-Hélène, CEA

Howard Brenda, CEH (UK)

Huet Christian, IRSN

Ikaheimonen Tarja, STUK (Fin)

Jacob Sophie, IRSN

Juhel Thierry, CEA

Kavasi Norbert, Japon

Kershaw Peter, CEFAS (UK)

Kesminiene Ausra, IARC

Kramer Gary, USA

Lallemand Jeannine, EDF

Lamart Stéphanie, NIH Bethesda (USA)

Laurent Gérard, EDF

Le Guen Bernard, EDF

Lebaron-Jacobs Laurence, CEA

Leggett Rich, ORNL (USA)

Lemaitre Nathalie, IRSN

Lenoir de Carlan Loic, CEA

Loyen Jeanne, IRSN

Luccioni Catherine, CNAM

Makovicka Libor, Univ. Franche Comté 
LISTE DES LECTEURS

Manduci Loredana, EAMEA

Marie Laurent, IRSN

Maubert Henri, CEA

Mehl-Auget Isabelle, ASN

Melintescu Anca-Mirela, Roumanie

Ménéchal Philippe, ASN

Ménétrier Florence, CEA

Métivier Henri, SFRP

Milliat Fabien, IRSN

Mitchell Peter, Univ Dublin (Irl)

Murith Christophe, BAG $(\mathrm{CH})$

Nies Harmut, AIEA (Monaco)

Niort Isabelle, Univ. Limoges

Organo Catherine, RPII (Irl)

Paquet François, IRSN

Paul Didier, CEA

Perrin Marie-Line, ASN

Perrin Anne, CRSSA

Petitot Fabrice, IRSN

Plazas Maria Cristina, Univ. Bogota (Col)

Pourcelot Laurent, IRSN

Provens Hélène, IRSN
Quesne Benoît, AREVA

Rannou Alain, IRSN

Rehel Jean-Luc, IRSN

Rennesson Malvina, ANDRA

Roue Amélie, CEA

Sabroux Jean-Christophe, IRSN

Schneider Thierry, CEPN

Siclet Françoise, EDF

Solatie Dina, STUK (Fin)

Steinmann Philippe, BAG (CH)

Tamponnet Christian, IRSN

Thierry-Chef Isabelle, IARC

Thomas David, NPL (UK)

Thomassin Alain, IRSN

Tirmarche Margot, IRSN

Truffert Hubert, AREVA

Vaillant Ludovic, CEPN

Van Iseghem Pierre, SCK/CEN (B)

Van Lauwe Aymeric, CEA

Verdun Francis, CHUV $(\mathrm{CH})$

Vivier Alain, CEA

Zankl Maria, Helmholtz (All) 